\title{
Wearable haptic anklets for gait and freezing improvement in Parkinson's disease: a proof-of-concept study
}

\author{
Simone Rossi ${ }^{1}$ (i) $\cdot$ Tommaso Lisini Baldi $^{2} \cdot$ Marco Aggravi $^{2} \cdot$ Monica Ulivelli $^{1} \cdot$ David Cioncoloni $^{3} \cdot$ Viola Niccolini $^{1}$. \\ Lorenzo Donati ${ }^{1}$ - Domenico Prattichizzo ${ }^{2}$
}

Received: 23 July 2019 / Accepted: 21 May 2020

(C) Fondazione Società Italiana di Neurologia 2020

\begin{abstract}
Background and objective In a proof-of-concept study, we aimed to verify whether the wearable haptic anklets, a device that delivers personalized suprathreshold alternating exteroceptive stimulation at the anklets on demand, may improve the quality of walking, including the freezing of gate (FOG), in idiopathic Parkinson's disease (PD) patients. The clinical relevance of the presented device as a walking pacemaker to compensate the disturbed locomotion through the generation of a more physiological internal walking rhythm should be verified in a dedicated clinical trial.

Methods We tested 15 patients diagnosed as idiopathic PD, during their regular treatment regimen. Patients were evaluated during walking with the device switched on and off, personalized at their most comfortable cadence. Stride velocity, variance, and length, as well as FOG episode duration during walking or turning of $180^{\circ}$, were quantified by an optical high-performance motion capture VICON system.

Results The alternating, rhythmic, sensory stimulation significantly improved either walking velocity or reduced inter-stride variance. Effects were more variable on stride length. The significant reduction of FOG episodes' duration correlated with clinical severity of scales rating gate and balance. No safety problems occurred.

Conclusions The WEARHAP-PD device, whose Technology Readiness Level (TRL) is 6, significantly improved some walking abilities (walking velocity and stride variance) and reduced the duration of FOG episodes in idiopathic PD patients. Unlike the traditional auditory and visual explicit cues that require the user's allocation of attention for correct functioning, the interaction of the patients with the surrounding environment was preserved, due to the likely implicit processing of haptic stimuli.
\end{abstract}

Keywords Parkinson's disease $\cdot$ Freezing of gate $\cdot$ Wearable robotics $\cdot$ Gait disturbances $\cdot$ Vibration

Simone Rossi and Tommaso Lisini Baldi contributed equally to this work.

Simone Rossi

Simone.rossi@unisi.it; rossisimo@unisi.it; https://www.Sibinlab.it

1 Department of Medicine, Surgery and Neuroscience, Unit of Neurology and Clinical Neurophysiology, Siena Brain Investigation and Neuromodulation Lab (Si-BIN Lab), University of Siena, Policlinico Le Scotte, Viale Bracci, I-53100 Siena, Italy

2 Department of Information Engineering and Mathematics Human Centered Robotics Group, SIRSLab, University of Siena, Siena, Italy

3 U.O.P. Professioni della Riabilitazione, Azienda Ospedaliera Universitaria Senese, Siena, Italy

\section{Introduction}

The alleviation of continuous and episodic gait disturbances, as instability, increased cadence, reduced stride length, and reduced velocity and freezing of gait (FOG) [1], represents a major therapeutic challenge, if not an unmet need, in Parkinson's disease (PD) patients. Indeed, these symptoms often remain unresponsive to the best possible dopaminergic medical [2] and surgical [3] treatments, even when other motor symptoms improve [2]. This worsens the patients' daily quality of life [4], increases the risk of falling and disability, and in turn increases the burden for healthcare systems.

Gait disturbances and FOG are usually positively sensitive to external rhythmic stimulations in most, but not all, patients: both auditory and visual cues can improve walking $[5,6]$ and reduce FOG [6]. Auditory cueing demonstrated significant improvement of cadence, stride length, and velocity [7, 8]. 
In contrast, visual cueing significantly improved stride length only [5]. However, such positive effects of auditory and visual cues on walking and FOG are counterbalanced by an underestimated drawback that is inherent to explicit cues: they require the constant allocation of patients' attention to cueing (i.e., if the patient does not listen to or look at the cue, the effect cannot take place). This required active cooperation of the user to the cue's functioning obviously obliges patients to restrict contacts and interactions with the surrounding environment in everyday life, thereby representing an additive potential dangerous factor.

We reasoned that the haptic channel could represent a suitable and safer alternative for external cueing, based on these two premises: (1) vibratory stimulation is effective in transiently improving cardinal motor signs in PD, including step parameters [9-12]; (2) it does not require allocation of attention as auditory or visual cues to be effectively processed. Moreover, we reasoned that (3) a bilateral, alternating, rhythmic stimulation at the ankles can be tuned to a given frequency for cueing a personalized comfortable walking cadence, even in healthy subjects; (4) in case of unpredictable FOG, the patient (or the care giver) can even activate on demand the device by an easily accessible remote Bluetooth control (i.e., a smartphone); (5) finally, haptic stimuli applied on lower limbs might directly target the activity of spinal centers for locomotion that are particularly sensitive to proprioceptive and exteroceptive inputs $[13,14]$, meanwhile being hardly responsive (if not very indirectly through polysynaptic circuitries) to auditory and visual cueing.

On these premises, we investigated whether the exploited wearable haptic vibrating anklets (WEARHAP-PD device), originally developed and patented (Patent WO2017199171) for providing a gait cadence to a subject $[15,16]$ and to remotely guiding blind people [17], might be safely used as a sensory cue helping to improve walking abilities - including FOG_-in PD patients.

\section{Subjects and methods}

\section{Experiment 1: Preparatory findings in healthy subjects}

Twelve healthy subjects ( 6 males; age range $18-65$ years; mean $39.7 \pm 13.6$, non statistically different from the PD patients group) were firstly evaluated in adapting their gait cadence to an external constant rhythm provided by the WEARHAP-PD device. This study group served to verify which could be the most appropriate cadence of alternating vibratory stimulation to be subsequently applied to Parkinsonian patients. We intended "the most appropriate" cadence of stimulation as the one at which subjects automatically align or modify their stride sequence to an external rhythm applied through alternating vibrations at the ankles.

Participants were provided with an Android phone and two haptic interfaces. A pressure sensor, connected to the master anklet, was positioned under the right heel to sample pressure data and extract stride duration, which were then transmitted via Bluetooth to the smartphone and logged. In all the trials, participants walked while wearing headphones reproducing white noise to avoid entrainment due to the sound of vibrations and external stimuli. For each participant, the experimental session started with a preliminary acquisition of selfpaced gait along a $200 \mathrm{~m}$ path, to record the user's most comfortable cadence. In the second trial, the haptic interfaces were activated at a random rhythm. The frequency was randomly selected in a range of $0-20 \%$ faster than the previously estimated user's comfortable stride duration. No additional instruction or hint was given to the participant. For the following $200 \mathrm{~m}$, we evaluated the strides duration measured by the master haptic interface, and for each stride, we extracted the stride duration error, defined as the difference between the current stride duration and the reference gait cycle.

\section{Experiment 2: Patients}

Fifteen idiopathic PD patients regularly followed as outpatients by at least 3 years at the local clinical institution were included in the study. Each subject fulfilled the UK Brain Bank Criteria [18] at the diagnosis. Exclusion criteria were the presence of troublesome dyskinesia (verified by objective examination and by a sum score less than 2 at the items for dyskinesia of the section IV of the revised version of the Unified Parkinson Disease Rating Scale (UPDRS)) [19], concomitant neurological disorders other than PD, intellectual impairment (verified by a standard neuropsychological screening including Mini Mental State Examination, Frontal Assessment Battery and Trail Making Test), lumbar back pain, and all other clinical conditions affecting gate.

\section{Standard protocol approvals, registrations, and patient consents}

The study was approved by the Local Institutional Ethics Committee, and each patient gave her/his written informed consent to participate. Authorization has been obtained for the consent to disclose any recognizable video material.

\section{Clinical evaluation}

Patients were evaluated during their regular therapeutic regimen, because we intended to test the device in a fully ecological situation, comparable with everyday life, and because previous studies failed to indicate significant differences of effects of sensory cues on walking parameters between on and off 
medication conditions [20]. Each patient underwent a complete neurological examination, including the part III of the revised version of the UPDRS [19], and the compilation of the new FOG questionnaire (NFOG-Q) [21] and of the Tinetti scale for gait and balance [22]. The new FOG is divided in three parts: the first one identifies if the patient is a freezer or not; the second and third parts rate the severity and the impact of the FOG, respectively. Their sum produces the final score.

The Tinetti scale evaluates balance and gait: it includes 16 items, the former 9 addressing balance during sitting, arising from a chair, during standing, turning on place, and during the Romberg's test. The latter 7 items explore gait, and more specifically the height, symmetry and continuity of strides, the trunk deviation the position, and the trajectory of hells. The maximal (worst) global score is 28. Clinical-demographic characteristics of patients are summarized in Table 1.

\section{The WERAHAP-PD device}

Tactile vibratory sensitivity is influenced by the spatial location on the body, by the distance between the stimulators, by the frequency of stimulation, and by the age of the user. Previous studies have demonstrated that vibration is better sensed on hairy skin due to its thickness and nerve depth and that vibrotactile stimuli are best detected in bony areas [23]. Due to the aforementioned considerations and since our aim was to design an intuitive and non-obtrusive device which could be easily worn, we concentrated on the exploitation of vibrotactile belts, to be worn on ankles.

From a technical point of view, each device is composed by two cylindrical vibro-motors, independently controlled via an external PC (or a smartphone app) using the Bluetooth communication protocol (see Fig. 1).

The communication is realized with an RN-42 Bluetooth antenna connected to a $3.3 \mathrm{~V}$ Arduino pro-mini

Table 1 Demographic and clinical characteristics of the 15 PD patients

\begin{tabular}{ll}
\hline Age & $61 \pm 7.3$ years \\
Disease duration & $9 \pm 4.9$ years \\
LEDD & Mean, $530 \pm 180$; range, $350-900 \mathrm{mg} / \mathrm{die}$ \\
UPDRS-I & $1.7 \pm 1.7$ \\
UPDRS-II & $14 \pm 7.3$ \\
UPDRS-III & $26 \pm 13.2$ \\
UPDRS-IV & $1.8 \pm 1.2$ (no troubling dyskinesia) \\
FOG questionnaire & $11 \pm 6.1$ \\
Tinetti (balance) & $11 \pm 3.4$ \\
Tinetti (gate) & $8 \pm 2.8$ \\
\hline
\end{tabular}

$L E D D$ levodopa equivalent daily dose

UPDRS Unified Parkinson Disease Rating Scale

$F O G$ freezing of gate microcontroller. The wireless connection baud rate is $57,600 \mathrm{bps}$. The microcontroller installed on the board is used to independently control the activation of each motor and receiving data from an external PC. Note that the proposed vibro-motors are controlled by applying a certain amount of voltage, which determines both frequency and amplitude. Thus, frequency and amplitude cannot be changed independently. Since the user's maximal sensitivity is achieved around 200-300 Hz [24], two Precision Microdrives Pico Vibe vibration motors are used to generate a $250 \mathrm{~Hz}$ vibration. The motors have a vibration frequency range of $100-280 \mathrm{~Hz}$, lag time of about $20 \mathrm{~ms}$, and rise and stop time of $35 \mathrm{~ms}$. The anklet guarantees about $4 \mathrm{~h}$ of battery life with one motor always turned on. Each device weighs about $90 \mathrm{~g}$.

\section{Tuning the WEARHAP-PD device}

The most comfortable walking velocity in healthy subjects aged 50-70 years is on average $1.3 \mathrm{~m} / \mathrm{s}$. Under normal conditions, each stride - calculated as the time elapsed from two consecutive heel supports of the same feet - has a length corresponding to about $80 \%$ of the subject's height. For example, in a person $1.7 \mathrm{~m}$ tall, the stride measures $1.35 \mathrm{~m}$ : this means that if the individual walks at $1.3 \mathrm{~m} / \mathrm{s}$, it will take $1.05 \mathrm{~s}$ to complete a stride.

The left/right alternating vibrotactile stimulus at the ankles is applied with an initial frequency according to the average walking speed of the patient. After having tuned the intensity of the stimulation well above the perceptive threshold, the timing of left/right alternating stimulation is individually tuned to the most comfortable walking speed, as subjectively reported by the patient and as previously verified in the experiment 1. In our sample of patients, these most comfortable velocities ranged from 1.6 to $1 \mathrm{~s}$ per step.

\section{Quantitative and qualitative walking assessment}

A motion caption system (Vicon Motion Systems Ltd., UK) was used for obtaining gait data regarding the walking patterns of the patients. Each user wore 39 retroreflective markers (Fig. 2), which were labeled using the software Nexus version 2.7 (Vicon Motion Systems Ltd., UK). With Nexus, we were able to record and identify the walking pattern of each user, with a precision on the position of the markers of $0.1 \mathrm{~mm}$. The trials took place in an available area of $12.20 \times 5.70 \mathrm{~m}$, where users were asked to walk in the middle, parallel to the long side. In our study, we considered three conditions for the evaluation: no-haptic suggestions mode, haptic-suggestions mode, and mixed mode. In the former condition, users walked from one side to the other of the room four times (two goes and two returns, ending the trial at its initial point), while no haptic suggestion was sent. In the haptic condition, the users were required to act as previously, but this time haptic stimuli, 

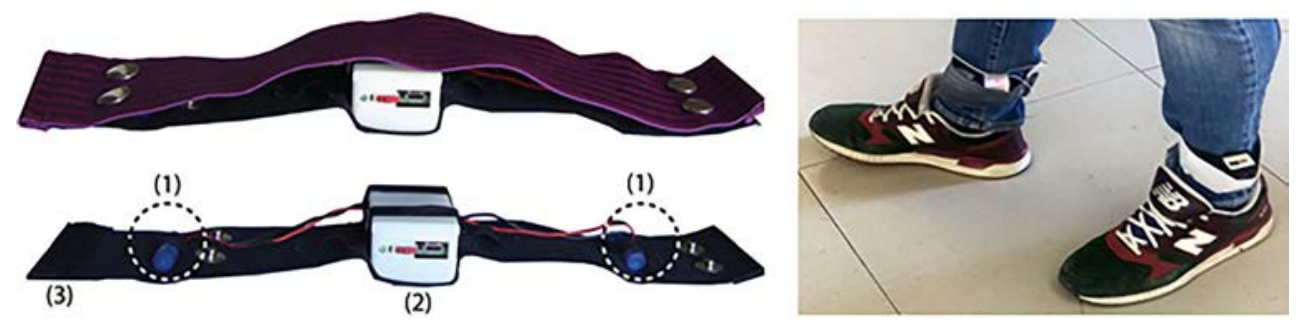

Fig. 1 The WEARHAP-PD device. The anklets are composed of two vibrating motors (1) attached to an elastic band (3). A Li-Ion battery and an Arduino board are in (2). When the device is engaged, its

provided by the WERAHAP-PD devices, were used to give a timing information on the gait. In the latter case, the number of trials (walking from one side to another and come back) was the same, but we used haptic stimuli in the first two walking and no cueing for the remaining two. Each user performed 12 walking trials (four per condition).

For each step, we considered the (a) elevation from the ground of the mean for the three markers composing the feet (Fig. 2) and (b) its trajectory (position in time) along the space for the all trial; in this way, we obtain a quantitative measure of how much each foot was going high and how long were the steps of each patient. Velocity and inter-stride variance were also computed. FOG time was evaluated as well, either when occurring unexpectedly during walking or when patients had to turn by 180 degrees at each corner of the room.

\section{Data availability statement}

All data, including those not published within the article, are available on request from any qualified investigator to the first two authors. Data will eventually be provided in anonymized form. Data will be shared by request.

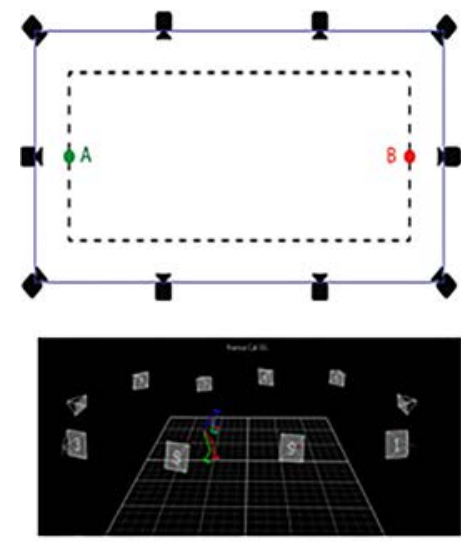

a

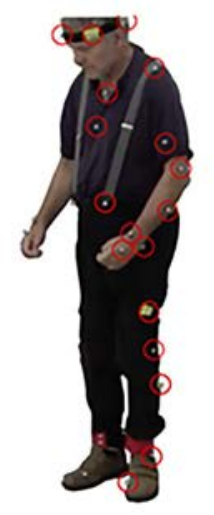

b

Fig. 2 a Available workspace $(12.20 \times 5.70 \mathrm{~m})$ with cameras and trackable workspace (dashed line) of $10 \times 4$ meters. Tracker software workspace shows the Vicon cameras and the patient walking from point A toward point B. b The subject is equipped with 39 retro reflective

motors vibrate at a frequency of $250 \mathrm{~Hz}$. The effective vibration frequency range is $100-280 \mathrm{~Hz}$ (the maximal human's sensitivity is achieved around $200-300 \mathrm{~Hz}$ )

\section{Results}

\section{Experiment 1: Preparatory findings in healthy subjects}

A Spearman's rank-order correlation was run to assess the relationship between the capability of subjects to automatically align the cadence with the proposed rhythm and the distance of the suggested cadence with respect to the user's most comfortable gait. Thus, we measured the capability in synchronization as the difference between the suggested cadence and the actual one of participants.

Analyses showed the relationship to be monotonic, as assessed by visual inspection of a scatterplot. Both variables (i.e., difference between the suggested and most comfortable cadence, real user's gait) were not normally distributed, as assessed by Shapiro-Wilk test $(p<.05)$, and there were no outliers. There was a statistically significant, strong negative correlation between the two variables, $r(12)=-0.907$, $p<0.0005$.

Thus, results showed that subjects automatically aligned to the walking pace only if the rhythm of the vibratory

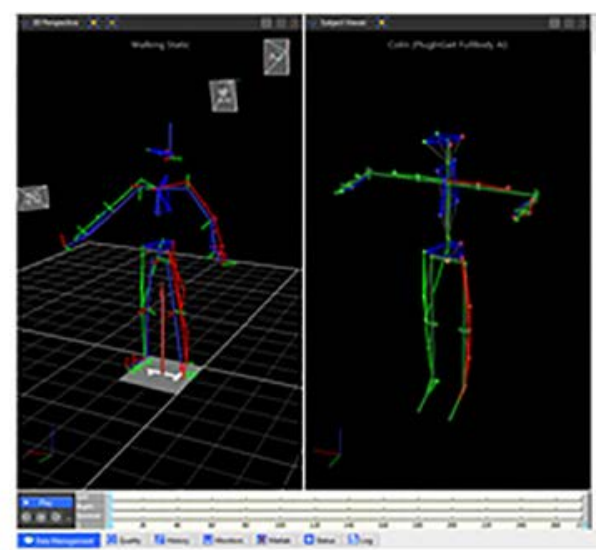

C

passive markers (red circles, only few are visible, due to the prospective), to enable the optical tracking system to recover its pose at each time step. The WEARHAP-PD device is worn at ankles. $\mathbf{c}$ The 3-D sensor-based reconstruction of the body 
stimulation was close to subjects' preferred walking cadence, while the influence of the vibration was negligible and did not affect the gait when the rhythm was far from the most comfortable one. This is a common behavior, extensively discussed in the literature for different types of cueing [25-28] and is the reason why we chose the "most comfortable cadence" in Parkinsonian patients.

\section{Experiment 2: Patients}

Table 1 summarizes clinical characteristics of patients, including current therapy. As interviewed at experimental debriefing, all the patients were comfortable with the device and subjectively reported no difficulty to use it. No particular safety concerns emerged, including the absence of any minor side effect.

\section{Stride kinematics}

Walking velocity Inspection of Vicon motion system video recordings was carried out with the WEARHAP-PD device switched on and off, for comparable epochs in the two

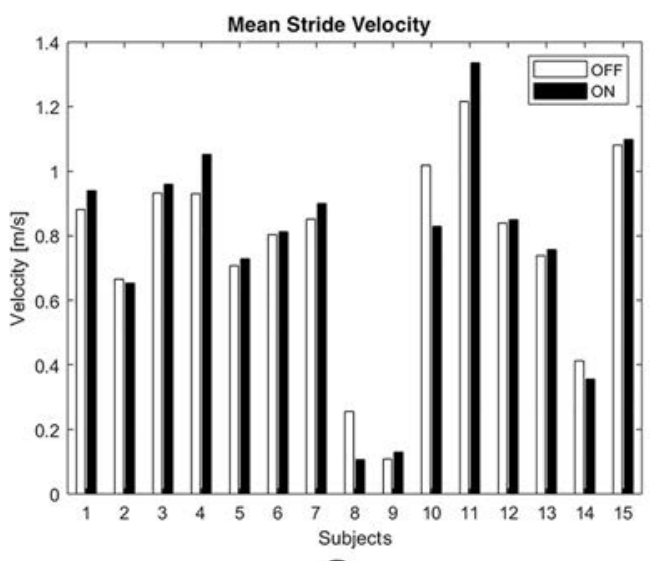

a

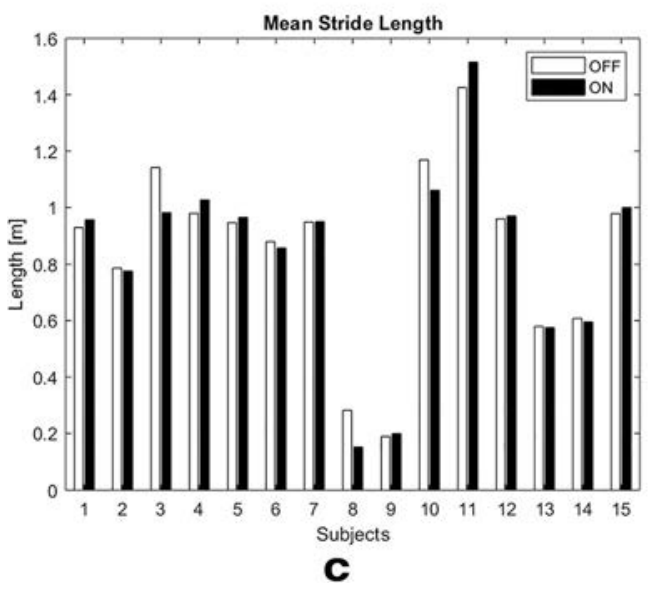

Fig. 3 In all panels, white and black bars identify results with the anklets switched off and on, respectively. Numbers on the $x$ axis identify single patients. a Mean stride velocity $(\mathrm{m} / \mathrm{s})$. b Stride standard deviation conditions. Stride velocity in $\mathrm{m} / \mathrm{s}$ increased in $11 / 15$ patients (mean increase 4.5\%; upper range 10\%) (Fig. 3a) when the device was switched on. In two patients (number $8,10)$, instead, the stride velocity decreased: at a closer inspection of clinical data, especially in relation to an unsatisfactory levodopa response, these patients underwent brain MRI during the study period that showed numerous ischemic lesions in the basal ganglia (that were not present at the time of diagnosis), possibly complicating the initial diagnosis of idiopathic PD. Thus, these data were excluded in the further statistical analysis.

Mean stride velocity data in the remaining 13 patients were normally distributed (Shapiro-Wilk test, $p=0.149$ ). The increase of velocity induced by the alternating vibratory stimulation was overall significant (paired sample Student $t$ test $=-$ 2.337, $p=0.038$ ). Such increase was even more significant after exclusion of the two other patients (numbers 2 and 14, see Fig. 3a) who were considered non-responder.

Stride length and height As shown in (Fig. 3c), effects of the WEARHAP-PD device on the stride length were variable: increase in 7 , decrease in 6 (including patients 8 and 10),
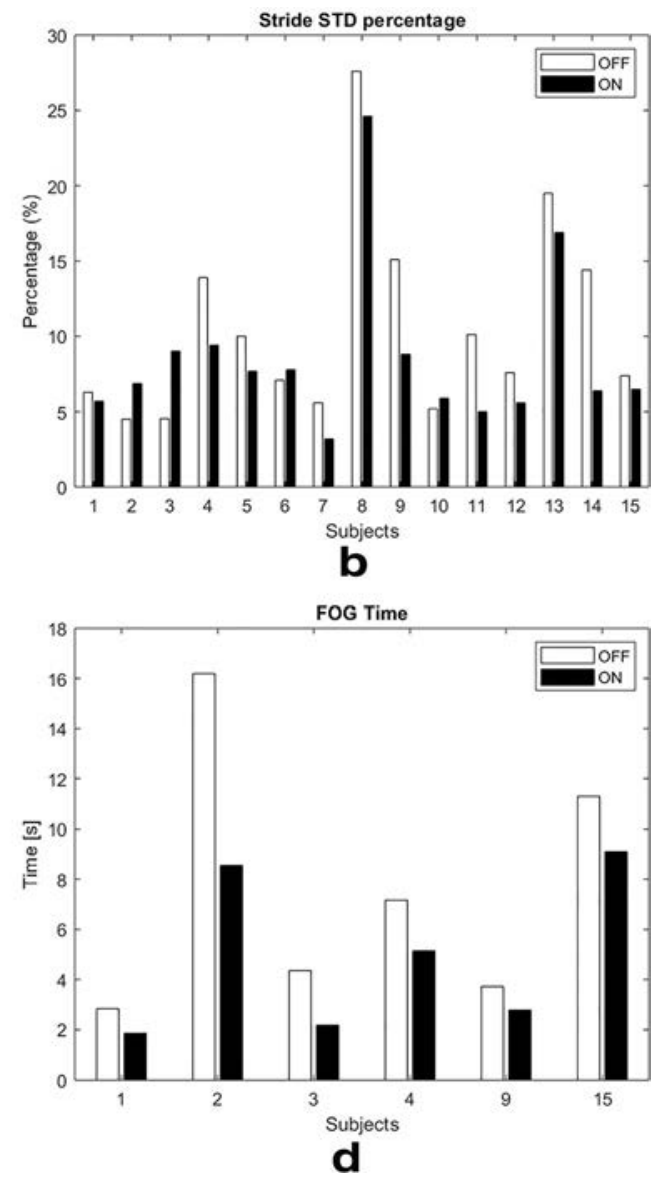

percentage (square root of the variance). c Mean stride length. d FOG time. In all panels, white bars indicate the devices switched off, and black bars indicate the devices switched on 
and no change in two. The lack of stride length increase in 8 patients (that, however, increased walking velocity as previously said) is simply consequent to the alignment to their most comfortable cadence as tuned by the WEARHAP-PD devices. Stride height did not change significantly with the device switched on (data not shown).

The two patients (8 and 10) with vascular lesions in the basal ganglia worsened stride length and height, besides velocity, with the device on, thus suggesting that vascular lesions might impair a correct supraspinal processing of the haptic cueing.

Inter-stride variance In normal subjects, the inter-stride variance is below $1-2 \%$, while in PD patients, it is increased due to the lack of rhythmicity [6]. The goal of the WEARHAP-PD device is to act as a walking pacemaker to compensate the disturbed locomotion through the generation of a new internal walking rhythm. To verify whether the device regulates inter-stride variance, in each patient, we measured the average length of left and right strides in every trial, with the device switched on and off. Figure $3 \mathrm{~b}$ shows that the inter-stride standard deviation (STD) with the device on is reduced by $10 \%$ on average, thus suggesting that the alternating haptic stimulation regularized stride length.

To properly verify the capability of our system, we took into consideration the reduction of STD percentage with the respect of the average stride length. Individual values are graphically depicted in Fig. 3b.

Paired sample Student $t$ tests were conducted to assess differences in walking with or without the vibrotactile cueing. Three tests were run in the sample: (1) for the entire patients' group, (2) after exclusion of subjects 8 and 10 (patients with vascular lesions), and (3) after further exclusion of patients 2 and 14 (non-responders for walking velocity improvement). It is worth pointing out that subjects 8 and 14 greatly improved their walking ability. All the tests showed a statistically significant decrease in inter-stride STD walking with the active anklets versus the off condition: test (1) $t(14)=2.319, p=0.039$; (2) $t(12)=2.188 p=0.048 p<0.05$; and (3) $t(10)=2.236$ $p=0.049<0.05$.

FOG time reduction Of the 15 patients, 6 had FOG. In each patient, the duration of each FOG episode was measured during the WEARAP-PD device switched on and off: as shown in Fig. $3 \mathrm{~d}$, the FOG duration decreased in all patients, ranging from 2.5 to $16.2 \mathrm{~s}$ with the devices in off and from 1.8 to $8.2 \mathrm{~s}$ with the devices switched on. This corresponds to a percentage improvement ranging 19.5-49.9\%. This difference was significant ( $p<0.05$, Student $t$ test), after verifying the normality of distribution of FOG episode durations (ShapiroWilk, $t=3.009, p=0.16>0.05)$.
Correlations There was a significant correlation between the severity of the overall Tinetti's clinical scale with the percentage reduction of FOG time (Pearson, $r=-0.679 ; p=0.05$ ), as well as with the Tinetti rating gate $(r=0.843 ; p=0.035)$.

To prove and validate results on FOG time reduction, a statistical analysis test was conducted on the data, analyzing the time in recovering from a FOG episode (dependent variable) under the two different conditions, vibrating anklets switched on or off (independent variable). Pearson's partial correlation was run to assess the relationship between rates in the NFOG questionnaire and percentage reduction of time of FOG episodes.

There was a linear relationship between the two variables. Both variables were normally distributed as assessed by Shapiro-Wilk test ( $p=0.880$ and $p=0.098$ for questionnaire answers and time percentage reduction, respectively). A bivariate Pearson's correlation showed a statistically significant linear relationship $(r=0.858, p=0.029)$.

\section{Discussion}

Traditional auditory and visual cues are defined as temporal or spatial stimuli able to regulate and facilitate repetitive movements, as walking, by providing an explicit motor target. They obviously require constant allocation of attention for their correct functioning, thereby distracting users from the surrounding environment. Previous pilot studies of alternating vibratory stimulation for walking improvement in PD used stimuli applied on the soles, time-locked with the foot stand on the ground [9, 12], with the aim to improve the defective proprioceptive feedback usually found in PD [29]. When positioned at the ankle, the vibrating device is in very close contact with the rigid surface of the leg bones, and therefore, vibrations are more likely to be perceived than when applied to the soles [9, 12]. Moreover, daily clinical practice indicates that sensory disturbances are usually more prominent in most distal limbs segments and that an overt or subclinical neuropathy is very common in PD, ranging from $16 \%$ if only large nervous fibers are considered to $56 \%$ if also small fibers are taken into account [30]. Thus, we reasoned that positioning the vibratory device at the ankles, rather than at the soles, will render more efficiently the processing of the haptic inputs in most of subjects.

Moreover, rather than improving foot stand-related proprioceptive feedback, the novel aim of WEARHAP-PD device is to provide the haptic cueing in a feed-forward mode, as a walking pacemaker tuned at the most comfortable subjective walking speed: indeed, manipulating in real time the dynamical environment during walking is commonly used to mechanically entrain gait patterns $[16,26]$. As such, the device can be easily tuned by patients or caregivers (via a smartphone app) according to their requirements in everyday life. Such non-continuous utilization of the device, together with the 
possibility to easily modify stimulation parameters, will minimize habituation to cueing in everyday life [6]. However, this aspect has been not specifically addressed in the current laboratory investigation, and it will require a prolonged use of the WEARHAP-PD device, in a domestic context.

Locomotion is a voluntarily initiated repetitive movement and then maintained by oscillating interneuronal pools located in the zona intermedia of the gray matter of the spinal cord at L2-L4 myelomers, called central pattern generators (CPG) [13]. CPGs may generate their oscillatory neural activity even when the spinal cord is lesioned rostrally [12]. CPGs that are silent at rest are under direct control from brainstem centers via reticulospinal neurons, which integrate afferent sensory feedback [31,32]: indeed, they are known to be extremely sensitive to large diameter sensory afferent fiber inputs, as those proprioceptive (neuromuscular spindles, Golgi tendon organs) and exteroceptive (tactile mechanoreceptors) ones continuously activated during walking [13]. The alternating suprathreshold haptic input generated by the WEARHAPPD device is likely to activate most of all large diameter sensory afferents channel mediated by the posterior columns of the spinal cord: as the stimulation is tuned according to "the most comfortable walking cadence", it is likely that the artificial afferent input might favor the reactivation of more physiological pattern of oscillatory neural activity of CPGs, either acting at segmental or suprasegmental levels. This might theoretically explain either the improvement of walking parameters or the improvement/resolving of the FOG.

Walking is a complex function, in which diffuse supraspinal networks involving basal ganglia, thalamus, supplementary motor area, and cerebellum are key structures in the functional loop implementing such rhythmical automatized movements [33, 34]. Basal ganglia obviously play a major and complex role in the pathophysiology of gait problems, including FOG, in PD patients [35]: one of their function is to correctly process sensory inputs [36,37]. An inadequate integration of sensory inputs at the striatum and a defective proprioceptive feedback in PD contributes to the alteration of sensorimotor integration and movement control [29, 38]. Functional neuroimaging investigations in PD patients have been obviously focused only on cortical and subcortical regional activity, and they point to an "imbalance between subcortical and cortical brain activation underlying the freezing episodes" [39]; moreover, they still do not offer univocal explanations of the effects of external cueing on neural processing $[6,40]$. It cannot be excluded that the alternating vibratory input generated by the WEARHAP-PD device might additionally improve basal ganglia sensory processing and, in turn, restore a more physiological cortical descending drive toward supraspinal walking centers (i.e., mesencephalic locomotor region, locus ceruleus, and reticular formation). The lack of effects of the WEARHAP-PD device in the two patients in which vascular basal ganglia lesions were detected would indirectly support this hypothesis, as the burden of white matter subcortical hyperintensities correlates with the development of FOG and its resistance to treatment [41]. Animal studies are required to definitely clarify neurophysiologically the relative weight of propriospinal or supraspinal mechanisms engaged by the WEARHAP-PD device alternating stimulation.

Whatever the neurophysiological mechanisms, current behavioral results are encouraging to look at the WEARHAPPD device as a cheap, safe, and easy to use wearable device for the improvement of some walking parameters in PD patients under regular pharmacological treatment. Moreover, if direct effects on CPGs would really mechanistically prevail, there could be room to look at the use of WEARHAP-PD devices even in patients with some degree of cognitive impairment, a common condition in PD patients with FOG that may reduce the success of external cueing based on stimulation of specific sensory channels as hearing or sight [42].

At a descriptive level, two patients in the current sample (number 8 and 10 of figures) showed vascular lesions of basal ganglia, poor response to levodopa, and highest scores to UPDRS-III items addressing bradykinesia and rigidity especially at lower limbs, as well as postural instability. Also in the other two patients who did not respond to the haptic cueing of the WEARHAP-PD device, bradykinesia and rigidity prevailed. These preliminary observations should be verified on larger samples that will help to verify which kind of Parkinsonian phenotypes are better responsive to the device.

\section{Limitations of the study}

While the small sample size of the study fits a proof-ofprinciple design addressing feasibility of the approach, safety, and acceptability of the device, it will need to be largely increased for a clinical trial. Such trial will benefit from a multicentric design, with gate and FOG measures taken outside the boundaries of the lab in a fully ecological domestic context, and possibly capitalizing by wearable sensors documenting quantitatively their occurrence.

The clinical trial should also address whether effects of the WEARHAP-PD device will change according to off and on states of patients and which patient's phenotype is the best candidate for using the device. For example, it is known that both age and PD can lead to a decline in haptic acuity [43, 44]; therefore, a longer disease duration in elderly patients might prevent the optimal functioning of the device.

Another limitation that generalizes to all other neuroimaging investigations in PD might be represented by the difficulty to causally demonstrate in humans the hypothesized effect on spinal CPGs.

Authors' contribution Simone Rossi: Study conception, patients' recruitment, supervision of data acquisition, and manuscript preparation. 
Tommaso Lisini Baldi: Device conception and manufacturing, data acquisition and analysis, and manuscript preparation.

Marco Aggravi: Device conception and manufacturing and data acquisition and analysis.

Monica Ulivelli: Patients' recruitment, clinical coordination, and critical revision of the manuscript.

David Cioncoloni: Data acquisition and organization.

Viola Niccolini: Data acquisition and analysis.

Lorenzo Donati: Data acquisition and analysis.

Domenico Prattichizzo: Device and study conception and critical review of the manuscript.

Funding information The research leading to these results has received funding from the European Union's Horizon 2020 research and innovation program - Societal Challenge 1 (DG CONNECT/H) under grant agreement n. 643644 of the project "ACANTO: A CyberphysicAl social NeTwOrk using robot friends" and under grant agreement $n^{\circ} 601165$ of the project "WEARHAP-WEARable HAPtics for humans and robots".

\section{Compliance with ethical standards}

Conflict of interest None.

Ethical approval None.

\section{References}

1. Nieuwboer A, Dom R, De Weerdt W, Desloovere K, Fieuws S, Broens-Kaucsik E (2001) Abnormalities of the spatiotemporal characteristics of gait at the onset of freezing in Parkinson's disease. Mov Disord 16:1066-1075

2. Nonnekes J, Snijders AH, Nutt JG, Deuscl G, Giladi N, Bloem BR (2015) Freezing of gait: a practical approach to management. Lancet Neurol 14:768-778

3. Abboud H, Genc G, Thompson NR, Oravivattanakul S, Alsallom F, Reyes D, Wilson K, Cerejo R, Yu XX, Floden D, Ahmed A, Gostkowski M, Ezzeldin A, Marouf H, Mansour OY, Machado A, Fernandez HH (2017) Predictors of functional and quality of life outcomes following deep brain stimulation surgery in parkinson's disease patients: disease, patient, and surgical factors. Parkinsons Dis 5609163

4. Muslimovic D, Post B, Speelman JD, Schmand B, de Haan RJ (2008) Determinants of disability and quality of life in mild to moderate Parkinson disease. Neurology 70:2241-2247

5. Spaulding SJ, Barber B, Colby M, Cormack B, Mick T, Jenkins ME (2013) Cueing and gait improvement among people with Parkinson's disease: a meta-analysis. Arch Phys Med Rehabil 94: $562-570$

6. Ginis P, Nackaerts E, Nieuwboer A, Heremans E (2018) Cueing for people with Parkinson's disease with freezing of gait: a narrative review of the state-of-the-art and novel perspectives. Ann Phys Rehabil Med 61:407-413

7. Thaut MH, McIntosh GC, Rice RR, Miller RA, Rathbun J, Brault JM (1996) Rhythmic auditory stimulation in gait training for Parkinson's disease patients. Mov Disord 11:193-200

8. Cochen Decock V, Dotov D, Damm L, Picot MC, Ihalainen P, Driss V, Geny C, Bardy B, Dalla Bella S. (2019) BeatPark: a new wearable device for gait auto-rehabilitation in Parkinson's disease delivering adapted musical stimulation. Mov Disord 34: (suppl 2) [abstract]
9. Novak P, Novak V (2006) Effect of step-synchronized vibration stimulation of soles on gait in Parkinson's disease: a pilot study. J Neuroeng Rehabil 3:9

10. King LK, Almeida QJ, Ahonen H (2009) Short-term effects of vibration therapy on motor impairments in Parkinson's disease. NeuroRehabilitation 25:297-306

11. De Nunzio AM, Grasso M, Nardone A, Godi M, Schieppati M (2010) Alternate rhythmic vibratory stimulation of trunk muscles affects walking cadence and velocity in Parkinson's disease. Clin Neurophysiol 121:240-247

12. Winfree KN, Pretzer-Aboff I, Hilgart D, Aggarwal R, Behari M, Agrawal SK (2013) The effect of step-synchronized vibration on patients with Parkinson's disease: case studies on subjects with freezing of gait or an implanted deep brain stimulator. IEEE Trans Neural Syst Rehabil Eng 5:806-811

13. Kuo AD (2002) The relative role of feedforward and feedback in the control of rhythmic movements. Mot Control 6:129-145

14. Dimitrijevic MR, Gerasimenko Y, Pinter MM (1998) Evidence for a spinal central pattern generator in humans. Ann N Y Acad Sci 860:360-376

15. Lisini Baldi T, Paolocci G, Prattichizzo D (2018) Human guidance: suggesting walking pace under manual and cognitive load. In EuroHaptics 2018: Haptics: science, technology, and applications, volume 10894, Pages 416-427, Pisa, Italy

16. Lisini Baldi T, Paolocci G, Barcelli D, Prattichizzo D (2020) Wearable haptics for remote social walking. IEEE Trans Haptics, 2020 (in press)

17. Scheggi S, Talarico A, Prattichizzo D (2014) A remote guidance system for blind and visually impaired people via vibrotactile haptic feedback. In 22nd Mediterranean conference on control and automation (pp. 20-23). IEEE

18. Hughes AJ, Daniel SE, Kilford L, Lees AJ (1992) Accuracy of clinical diagnosis of idiopathic Parkinson's disease. a clinicopathological study of 100 cases. J Neurol Neurosurg Psychiatry 55:181-184

19. Goetz CG, Tilley BC, Shaftman SR, Stebbins GT, Fahn S, Martinez-Martin P, Poewe W, Sampaio C, Stern MB, Dodel R, Dubois B, Holloway R, Jankovic J, Kulisevsky J, Lang AE, Lees A, Leurgans S, LeWitt PA, Nyenhuis D, Olanow CW, Rascol O, Schrag A, Teresi JA, van Hilten JJ, LaPelle N, Movement Disorder Society UPDRS Revision Task Force (2008) Movement Disorder Society-sponsored revision of the unified Parkinson's disease rating scale (MDS-UPDRS): scale presentation and clinimetric testing results. Mov Disord 23:2129-2170

20. Barthel C, Nonnekes J, van Helvert M, Haan R, Janssen A, Delval A, Weerdesteyn V, Debû B, van Wezel R, Bloem BR, Ferraye MU (2018) The laser shoes. A new ambulatory device to alleviate freezing of gait in Parkinson disease. Neurology 90:e164-e171

21. Nieuwboer A, Rochester L, Herman T, Vandenberghe W, Emil GE, Thomaes T, Giladi N (2009) Reliability of the new freezing of gait questionnaire: agreement between patients with Parkinson's disease and their carers. Gait Posture 30:459-463

22. Tinetti ME, Williams TF, Mayewski R (1986) Tinetti balance assessment tool. Fall risk index for elderly patients based on number of chronic dis- abilities. Am J Med 80:429-434

23. Gemperle F, Hirsch T, Goode A, Pearce J, Siewiorek D, Smailigic A (2003) "Wearable vibro-tactile display," Corneige Mellon University

24. Riener A (2011) Sensor-actuator supported implicit interaction in driver assistance systems, S. H. Et al., Ed. Springer, vol. 10

25. Roerdink M, Bank PJ, Peper CL, Beek PJ (2011) Walking to the beat of different drums: practical implications for the use of acoustic rhythms in gait rehabilitation. Gait Posture 33:690-694

26. Ahn J, Hogan N (2012) Walking is not like reaching: evidence from periodic mechanical perturbations. PLoS One 7:e31767 
27. Leman M, Moelants D, Varewyck M, Styns F, van Noorden L, Martens JP (2013) Activating and relaxing music entrains the speed of beat synchronized walking. PLoS One 8:e67932

28. Damm L, Varoqui D, De Cock VC, Dalla Bella S, Bardy B (2019) Why do we move to the beat? A multi-scale approach, from physical principles to brain dynamics. Neurosci Biobehav Rev 112:553584. https://doi.org/10.1016/j.neubiorev.2019.12.024

29. Abbruzzese G, Berardelli A (2003) Sensorimotor integration in movement disorders. Mov Disord 18:231-240

30. Zis P, Grünewald RA, Chaudhuri RK, Hadjivassiliou M (2017) Peripheral neuropathy in idiopathic Parkinson's disease: a systematic review. J Neurol Sci 378:204-209

31. Zehr EP, Duysens J (2004) Regulation of arm and leg movement during human locomotion. Neuroscientist 10:347-361

32. Nielsen JB (2003) How we walk: central control of muscle activity during human walking. Neuroscientist 9:195-204

33. Kotz SA, Schwartze M (2010) Cortical speech processing unplugged: a timely subcortico-cortical framework. Trends Cogn Sci 14:392-399

34. Kotz SA, Schwartze M (2011) Differential input of the supplementary motor area to a dedicated temporal processing network: functional and clinical implications. Front Integr Neurosci 5:86

35. Nutt JG, Bloem BR, Giladi N, Hallett M, Horak FB, Nieuwboer A (2011) Freezing of gait: moving forward on a mysterious clinical phenomenon. Lancet Neurol 10:734-744

36. Boecker H, Ceballos-Baumann A, Bartenstein P, Weindl A, Siebner HR, Fassbender T, Munz F, Schwaiger M, Conrad B (1999) Sensory processing in Parkinson's and Huntington's disease: investigations with 3D H(2)(15)O-PET. Brain 122:16511665
37. Ulivelli M, Rossi S, Pasqualetti P, Rossini PM, Ghiglieri O, Passero S, Battistini N (1999) Time course of frontal somatosensory evoked potentials relation to L-dopa plasma levels and motor performance in PD. Neurology 53:1451-1457

38. Lewis GN, Byblow WD (2002) Altered sensorimotor integration in Parkinson's disease. Brain 125:2089-2099

39. Fasano A, Herman T, Tessitore A, Strafella AP, Bohnen NI (2015) Neuroimaging of freezing of gait. J Park Dis 5:241-254

40. Shik ML, Severin FV, Orlovsky GN (1996) Control of walking and running by means of electrical stimulation of the mid-brain. Biophysics 11:756-765

41. Chung SJ, Lee YH, Yoo HS, Oh JS, Kim JS, Ye BS, Sohn YH, Lee PH (2019) White matter hyperintensities as a predictor of freezing of gate in Parkinon's disease. Parkinsonism Relat Disord 66:105109

42. Nonnekes J, Ružicka E, Nieuwboer A, Hallett M, Fasano A, Bloem BR (2019) Comopensation strategies for gait impairment in Parkinson diseae. JAMA Neurol 76:718-725

43. Conte A, Khan N, Defazio G, Rothwell JC, Berardelli A (2013) Pathophysiology of somatosensory abnormalities in Parkinson disease. Nat Rev Neurol 9:687-697

44. Konczak J, Sciutti A, Avanzino L, Squeri V, Gori M, Masia L, Abbruzzese G, Sandini G (2012) Parkinson's disease accelerates age-related decline in haptic perception by altering somatosensory integration. Brain 135:3371-3379 